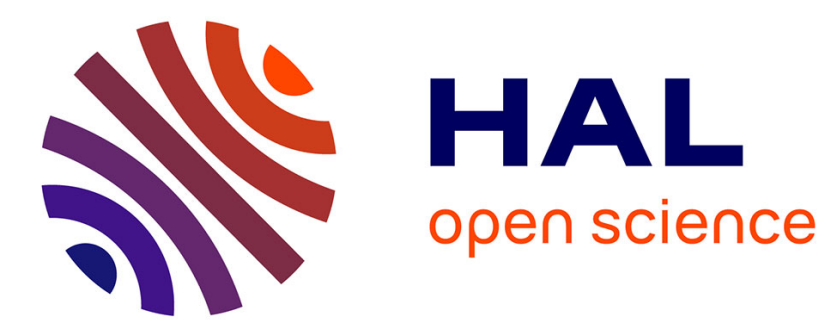

\title{
L'Espace interstitiel, lieu des possibles. Le futur selon Volodine
}

Khalil Khalsi

\section{To cite this version:}

Khalil Khalsi. L'Espace interstitiel, lieu des possibles. Le futur selon Volodine. Contemporary French and Francophone Studies, 2017, 21 (3), pp.329-336. 10.1080/17409292.2017.1403150 . hal-03118583

\section{HAL Id: hal-03118583 \\ https://hal.science/hal-03118583}

Submitted on 1 Apr 2021

HAL is a multi-disciplinary open access archive for the deposit and dissemination of scientific research documents, whether they are published or not. The documents may come from teaching and research institutions in France or abroad, or from public or private research centers.
L'archive ouverte pluridisciplinaire HAL, est destinée au dépôt et à la diffusion de documents scientifiques de niveau recherche, publiés ou non, émanant des établissements d'enseignement et de recherche français ou étrangers, des laboratoires publics ou privés. 
Ce papier est le brouillon final soumis à l'éditeur. Pour citer cet article, merci de vous référer à la version officielle publiée par Taylor \& Francis :

CONTEMPORARY FRENCH AND FRANCOPHONE STUDIES, 2017 VOL. 21, NO. 3, 329336 https://doi.org/10.1080/17409292.2017.1403150

\section{Khalil Khalsi \\ Université de Montréal \\ Université de la Sorbonne Nouvelle \\ L'espace interstitiel, lieu des possibles. \\ Le futur selon Volodine}

\section{Résumé :}

Antoine Volodine est le porte-parole d'une communauté fictive d'auteurs emprisonnés sous haute surveillance. Ces derniers mettent en réseau leurs voix, leurs récits et leurs identités, créant ainsi la cartographie d'un monde dystopique, où le sens circule à travers ce que je conceptualise comme un espace interstitiel. Zone de passage et de transition, il s'agit d'un espace d'individuation, que je propose de mettre en évidence à partir du Port intérieur (Minuit, 1995), dont le héros, avatar de l'individu post-moderne, ne paraît plus certain de son statut de «personne » vis-à-vis d'une réalité qui tend à l'objectiver et à le pousser à la disparition et à l'impersonnalisation (Rabaté, Esposito). Désincorporé, le personnage volodinien entre dans une zone d'énonciation impossible, où il ne peut assumer son « je » vis-à-vis d'un «tu », se laissant alors énoncer par l'esprit du lieu, ce qui déstabilise son ego pour n'en faire qu'une dimension corrélative à l'espace-temps. Cette prise de conscience douloureuse, doublée d'une renonciation au futur, ouvre une dialectique des contraires à travers laquelle le personnage se laisse individuer de manière fluide au-delà de l'absolue catégorisation identitaire (Serres, Agier) ; toutefois, dans cet état d'entre-deux, s'enclenche un processus transformationnel qui va dans le sens d'une révolution et qui révèle alors l'espace interstitiel comme un lieu d'utopie (Jameson), porteur d'un élan vers le futur et du germe d'une régénération collective.

Mots-clés : post-exotisme, espace interstitiel, disparition, individuation, personne, utopie. 
Antoine Volodine a édifié son œuvre, au fur et à mesure des textes, selon une cosmogonie de sa propre invention, celle du courant $\mathrm{du}$ « post-exotisme ». Cette étiquette, sur le plan éditorial, légitime l'œuvre volodinienne et la différencie des autres systèmes littéraires, tout en instituant un monde fictionnel par l'invention d'un ordre, d'une préhistoire et d'un devenir propre, devenir aussi incertain soit-il, entretenant un lien dystopique avec le monde réel. Faut-il rappeler qu'Antoine Volodine n'est que le pseudonyme d'un auteur qui s'incarne dans notre réalité, à la limite d'un univers autre dont il est le chamane, davantage le traducteur, le décrypteur, le prophète que le créateur ; il nous transmet la parole d'une communauté de « sur-narrateurs », tous enfermés dans un « quartier de haute sécurité » (Volodine, Le post-exotisme en dix leçons, leçon onze 10) qui les isole de tout, les réprime et les neutralise, pour avoir été ces guerriers égalitaristes dont la révolution mondiale a échoué à renverser le système capitaliste en place. Le post-exotisme est une parole de vaincus ; en ce sens, il n'offre a priori aucun espoir pour aucun des mondes possibles. Dès lors, il pourrait légitimement se placer dans l'héritage du Nouveau Roman (certains romans de Volodine ont d'ailleurs vu le jour chez Minuit, bastion de Sarraute et Co.), en tant que porteur d'une conscience post-traumatique européenne laquelle, certes, s'ancre dans un « $\mathrm{XX}^{\mathrm{e}}$ siècle malheureux » (Volodine, « Écrire en français une littérature étrangère »), mais c'est un siècle où le champ supposément visible de la souffrance s'élargit à la planète tout entière : combattants égalitaristes, les personnages volodiniens revendiquent — « [p]ar sympathie idéologique, par délégation poétique, par empathie » (Volodine, « À la frange du réel » 395) — une mémoire collective qu'ils partagent avec tous les peuples opprimés. Par réflexivité face à une déconsidération de l'Autre paradoxalement caractéristique du globalisme post-1989, le post-exotisme accomplit un décentrement des champs de vision, de pouvoir et d'énonciation, de quoi re-subjectiver l'Autre et amorcer la possibilité d'une rencontre et d'une communautarisation qui, quoique globale, va à l'encontre du mondialisme. C'est ce paradoxe que je propose d'élucider à travers la mise en évidence d'une certaine pensée de l'espace interstitiel, lieu de jonction-disjonction par où circule le sens et qui porte le potentiel métamorphique du collectif.

\section{De la disparition de la personne : concepts de base}

Dominique Rabaté identifie le paradigme du disparaître et du silence comme forme ultime d'une certaine fatigue civilisationnelle miroitée par la littérature hexagonale. Si le critique français voit

dans ce fantasme la fuite hors d'un «monde de traçabilité généralisée » (22), il pointe vers un 
paradoxe juridique puissant, que le philosophe Roberto Esposito explicite par « le dispositif de la personne » (9). Que le sujet occidental désire exister sans traces en raison de son omnivisibilité est le signe d'une désincarnation due à sa sur-personnalisation ; il prend conscience de son impossibilité d'exister en tant que personne en dehors du dispositif. C'est alors qu'il se rend compte de n'avoir jamais été qu'un « il », qu'une troisième personne, donc qu'une « non-personne » au sens de Benveniste (Esposito 106). De plus, sa non-personnalité tient au fait d'avoir performé sa personne au point que son masque, sa persona, est devenu ce qui le caractérise en tant qu'individu.

Ainsi donc, le propre de la personne post-moderne/post-exotique, c'est de n'en être plus une, si on lie la question du statut de la personne à un concept plus phénoménologique : l'individuation, cette capacité de l'individu à se construire en tant que tel. À cet égard, la notion de "personne », en tant que construction juridique socio-historique, s'oppose à celle d'« individu », supposée moins objectivante. Sachant que l'individuation est corrélative à l'écoumène, Cynthia Fleury la dit perdue de vue dans une époque où l'idée de cité, au sens politique du terme, est étiolée (18). Il faut toutefois distinguer cette errance, d'ordre idéologique, de celle, plutôt physique et psychique, à laquelle s'adonnent les personnages post-exotiques. Tandis que l'homme dans sa cité errante, pris dans l'engrenage du dispositif, croit jouir du statut de personne - ce qui en fait une non-personne —, le personnage post-exotique prend acte de cette non-personnalité pour errer et s'impersonnaliser hors de la cité, en l'occurrence occidentale. Or, le sujet volodinien, ainsi libéré des balises de son milieu d'origine et de la fiction de l'absolue catégorisation identitaire, semble constater une distance qui le sépare de l'Autre, ce qui compromettrait toute possibilité d'individuation. Dans la philosophie volodinienne, le « je » hésite à se dire, et encore moins à (se) désigner (vis-à-vis d')un « tu ». Comment donc l'individuation, ainsi que la collectivisation qu'elle induirait, pourraientelles s'envisager en dehors du cadre énonciatif?

\section{Cogito de l'impersonne}

À cet égard, le jeu pronominal est intéressant à analyser dans le roman Le Port intérieur, où le nom « Breughel » désigne l'instance narratrice qui se présente sous différents pronoms, souvenirs, rêves et masques, y compris celui de son ennemi, qu'il baptise Kotter. «Dupont aurait convenu aussi bien », confie ce dernier, ajoutant : «Mon nom n'a aucune importance. » (65) Bien qu'acceptant de se laisser attribuer un nom, Kotter fait allusion à un état d'anonymat primordial qui déshabilite son statut de personne. Or, tout au long du récit du transit de Kotter vers Macau, le 
point de focalisation oscille entre « Kotter » (repris par le pronom « il ») et « on », renvoyant à une conscience plus diffuse, plus neutre et peut-être plus intérieure, propre au cogito qui s'énonce et dont les fulgurances impersonnelles renvoient à un plan de conscience volontiers centrifuge. Entre l'assertion « On voit tout » et la suivante, « Kotter regarde », le décalage est manifeste ; d'un côté, un on aussi neutre qu'omniprésent (dénué de regard : on voit) et, de l'autre, un Kotter distancié, d'où fuit le regard, rendu hermétique. Le paradoxe est là : tandis qu'on, malgré sa neutralité, se laisse percevoir par un certain cogito, $i l$ regarde sans rapport d'identification visuelle qui puisse le définir en retour.

Ce brouillage énonciatif et identitaire est causé par la mise en exil, notamment à Macao, « lieu d'exil idéal » où l'« on va se cacher et [se] dissoudre» (Volodine, « Macao, l'entre-deuxmonde »). La mort, symbolique, s'accomplit par une sortie de l'environnement et du cadre juridique d'origine (on parle bien du « droit de dire adieu à tout, d'habiter ailleurs »), en se mettant hors-champ. On meurt de n'être pas vu. La neutralité du pronom on, renvoyant à l'impersonnalité revendiquée de l'énonciateur, s'impose vis-à-vis d'une culture autre, qui lui est "indifférente » (Jullien $)^{1}$. Sentiment bien ambivalent que celui qu'éprouve le personnage volodinien, entre cette « impression immédiate [d'être] en terre d'accueil » («Macao... ») et, parallèlement, l'incapacité d'établir le contact avec les autochtones qui « affectent de ne pas croire aux preuves de son existence » (Port... 36). En l'absence d'interaction avec le dehors, ce dernier finit par recouvrir l'existence du personnage qui semble alors s'y dissoudre. Par conséquent, ce n'est pas une quelconque entité subjective qui se trouve accueillie en terre étrangère, mais quelque chose d'indéfini, d'inorganique, de translucide qui retrouve naturellement « sa place » (« Macao... ») dans l'esprit du lieu. Tandis que l'ego « avance vers le zéro » (Port... 34), l'énonciateur impersonnel se laisse énoncer par le lieu auquel il se confond, un il y a tout à fait neutre, « hors conscience » (Esposito 130).

La neutralité identitaire correspond à une suspension de soi pour une meilleure identification avec ce quelque chose dont le concept foucaldien de bios, la « vie », serait éventuellement l'expression en tant qu'objet principal du (bio-)pouvoir (Esposito 136). Lors de cette rencontre authentique et absolue avec l'impersonnel, tombent les rapports de pouvoir et, auparavant, toute possibilité de masque, de persona, d'ego au sens catégorique et essentialiste. En ce sens, l'impersonnalisation, qui n'est en rien une dépersonnalisation, est une invalidation du concept même d'« identité », cette « chose » qui n'existe pas en soi (Agier 131). Ainsi, délesté de cette part de 
soi la plus dé-subjectivitée, l'exilé post-exotique se retrouve sur le terrain d'une rencontre impossible, qui renforce son incapacité à dire « je ». Par ailleurs, son deuil identitaire, qui est un deuil du passé, se double d'un effondrement du futur vers lequel portait son ancienne (non-)personne.

\section{L'ego à l'épreuve de l'espace interstitiel}

«L'affaire du deuil c'est l'avenir, c'est le temps qui se déroule désormais devant soi, et sans l'autre », (Fleury 66) — et sans soi, ajouterai-je. Plein de la pensée de Gloria, aimée et perdue, le deuil de Breughel s'exprime dans un état de déchéance, déserté de toute certitude du futur, donc de tout sens de la temporalité : «Breughel habitait désormais un temps indistinct [...]» (Port... 19). Il vit dans une cellule au fond d'une venelle, coupé du monde, une stase finale qui le maintient entre être et néant. Il s'agit là d'un état interstitiel où se dissémine l'individualité du personnage, entre laisser-aller et désir de survie, idées noires et souvenirs lumineux, déchéance et rêverie. Toutefois, le personnage se laisse progressivement esquisser tout au long de ces mouvements et contremouvements, essentiellement par une conjugaison des contraires, comme ce mouvement d'affirmation-négation : « Au-delà de cette ligne tu existes, tu n’existes pas. » (55) Cette double injonction, loin de clouer le $t u$ à un quelconque horizon de présence ou d'effacement, le saisit dans un prisme d'existence quasi-quantique, autrement plus fluide, où les contraires ne s'annulent pas, ni même se complémentent, mais se superposent pour ouvrir une nouvelle dimension d'existence, dont l'infini est le résultat de l'équation. Et pour qu'elle soit infinie, cette dimension interstitielle doit l'être tout autant que le temps, l'espace et l'ego qui la composent - temps, espace et ego infinis.

Ce principe de non-opposition des contraires tient, entre autres, des spiritualités orientales dont le post-exotisme fait un motif esthétique. Laïcisée, cette conciliation des contraires relève d'une mystique oxymorique, consistant en leur indifférenciation, de quoi ouvrir une interaction et une

continuité entre les opposés, entre le oui et le non, entre la mort et la vie. À cette ouverture, correspond une représentation cyclique du temps à même de faire circuler le sens par sa perpétuelle régénération. Cette liminalité est représentée chez les Tibétains par le Bardo Thödol, état intermédiaire entre la vie et la mort où l'âme est sommée de se dissoudre dans la corporation divine sans quoi, elle se réincarne. Les personnages post-exotiques, inspirés par Le Livre des morts tibétain, font souvent l'expérience de cet entre-deux, lieu de transit de l'âme qui y projette sa propre conscience. Stase insaisissable, cet espace non-objectif demeure de l'ordre de l'expérience. Michel 
Serres le décrit comme un espace intraduisible, donc impossible à verbaliser et uniquement expérimentable par l'affect. Il l'appelle « espace blanc» car contenant toutes les couleurs lesquelles, par leur différenciation, composent le spectre du neutre. L'individualité affirmée précède ou suit cet espace, zone de transit où le passager « n'est encore ni l'un ni l'autre et, devient peut-être, déjà, l'un et l'autre » (Serres 24). Certes, Breughel est perclus dans cet espace interstitiel, mais le lecteur peut déjà voir s'annoncer, par bribes, entre mouvements d'affirmation et de négation, « le fantôme d'un troisième homme » (Serres 29), qui ne se réalisera sûrement pas, mais qui fait signe vers une possibilité de régénération qu'entérinera, en l'occurrence, la lutte de Breughel contre Kotter et l'incorporation de ce dernier.

Cette logique de l'entre-deux miroite « une réalité faite de mondes multiples, illusoires et parallèles » (Volodine, Le Post-exotisme... 75), en ouvrant des isthmes entre les éléments antagoniques afin de régénérer le mouvement. L'ego en apparaît comme une dimension de l'espacetemps, lequel semble s'abolir lorsque l'individu expérimente ce que j'appellerai donc l'espace interstitiel. La distance se dissout, (l'on) n'existe plus que (dans) le pli. Ce pli, s'il ne s'agissait pas de la venelle où Breughel se laisse aller à sa déchéance, près du port intérieur de Macau, pourrait être figuré par ce train de Bardo or not Bardo (2004) qui circule à toute allure et sans arrêt à travers des contrées hétérogènes, que le pli traverse, dispose et recompose dans une temporalité onirique.

Le pli, nous dit Michel Serres, est un «germe de forme » (48) : à partir de ce pli prend place une réintégration cosmologique qui s'achemine vers une résolution de l'économie narrative, voire une révolution. Ce moment-là, qui survient avec une sortie du pli (à moins que le pli ne s'ouvre au monde et l'englobe), est celui où Kotter, se battant avec Breughel, «per[d] son identité » (177) et devient ce dernier, comme s'il n'avait jamais existé. Kotter se sera laissé entraîner dans le pli dont Breughel sort triomphant, certain de son je final — « Je me relevai » (179) —, issu d'une superposition identitaire entre un Breughel impersonnifié et un Kotter dominant qui se sera laissé incorporer.

\section{Entre être et néant, le lieu des possibles}

Il faut revenir à la question du neutre, ainsi qu'y répond Esposito, pour comprendre ce processus transformationnel : 
" [It] is an experience in which we lose the distinction between being and nothingness, night and day, life and death: it is a nothingness that continues to be; a day that is swallowed up in the darkness; a death that is prolonged into life. » (Esposito 129)

L'espace interstitiel est le lieu d'intersection de l'être et du néant où l'impersonne se trouve ; et c'est précisément dans cette stase-là, lorsque l'individu est incapable de dire «je », que le contact est possible. Que ce soit avec la Chine, terre d'ultime altérité autant pour Volodine que pour Breughel (terre où l'on n'existe pas), ou avec Kotter, le meurtrier (celui donc qui nous apporte la mort), il s'agit de prendre le « détour» de l'Autre afin de revenir sur soi, plutôt qu'à soi, comme à une nouvelle personne. C'est « sortir de la contingence de son esprit, ou prendre du recul dans son esprit, en passant par l'épreuve d'une pensée extérieure » et en renonçant, pour un temps, à « ce “je" qui dit si superbement : “je pense” » (Jullien 5). C'est, d'un côté, accepter que le cogito ne soit pas l'apanage de l'être, mais qu'il soit aussi celui du néant, de l'impensé, du trou noir. C'est renoncer à penser pour se laisser impenser et repenser, en s'abandonnant et se mettant à disposition d'une énonciation via le dissemblable.

Que Breughel arrive à bout de Kotter au point de la confusion et de l'indifférenciation, c'est bien le signe que l'espace interstitiel est le lieu d'une « transgressivité en acte » (Westphal 121). D'être proprement virtuel, cet espace est celui des possibles, malgré la conscience malheureuse du post-exotisme, dont la rhétorique est cependant celle de la résistance et de la dissidence (bien que vouée à l'échec) ; ceci ferait voir l'espace interstitiel comme un antidispositif impersonnel, tronquant le dispositif de la personne (le pouvoir supérieur, en l'occurrence le capitalisme) pour y ouvrir des lieux de passage, de transition et de métamorphose. Le moteur de cette dissidence, c'est la vie qui, parasitique, continue d'être portée d'individu en individu, d'impersonne en impersonne, en dessous des masques et des visages.

De là ressort l'aspect collectif de l'espace interstitiel en tant que lieu d'utopie, de métamorphose, de possible. Cet « échangeur » (Serres 28) est un lieu de passage, de circulation et de métaphore ${ }^{2}$, puissamment investi par les personnages volodiniens lorsqu'ils connectent leurs âmes, leurs identités et leurs rêves les uns aux autres ${ }^{3}$, pour aller et venir en toute liberté dans cet espace collectif qui ne serait possible sans le recul impersonnel. Pour Serres, cet espace existe déjà ; il s'agit du virtuel, dont le post-exotisme n'est aucunement l'allégorie, au contraire : le virtuel est précisément investi par le pouvoir, qui brouille le frontière privé-public laquelle se trouve, dès lors, sans cesse renégociée et reconfigurée - dans ces mouvements, apparaît l'interstitiel utopique. Et 
lorsque F. Jameson accuse la mondialisation de s'être approprié la « rhétorique de décentralisation » (Le désir nommé utopie 363), c'est bien pour mettre en évidence la nécessité quasi-naturelle d'ouvrir des « enclaves » utopiques, dans le tissu même de la dystopie totalitariste. C'est ce dont l'espace interstitiel volodinien me semble être l'expression futuriste : dans ce monde post-apocalyptique, les minorités culturelles sont dissoutes dans le magma du globalisme, guère plus présentes que d'en bas, en lutte, sous forme de survivance, déléguée dans le potentiel de dissidence.

Le post-exotisme opère une transformation paradigmatique, faisant apparaître la conscience planétaire comme malheureuse, certes, mais constellée d'utopies. À plus d'un égard, ce principe ressemble à la « méthode utopique » que propose Jameson pour s'acheminer vers un « alternate utopian future » («Utopia as Method » 42), méthode qui consiste à opérer une parallaxe pour inverser les contrastes, expressément renforcés, afin de déceler les possibilités de métamorphose et les germes du futur. Ce que Serres appelle «l'espace blanc», que Volodine voit comme un « espace noir », que H. Bhabha a baptisé «third space », etc., et que je propose de conceptualiser à partir du post-exotisme comme l'« espace interstitiel », c'est finalement le lieu du possible qui s'énonce là où s'opère la rencontre, où s'ouvre « l'écart » (Jullien), à partir duquel les forces vives apposent une résistance souterraine, dans le corps même du monde trop rapidement vu comme hybridé le consumérisme en trompe-l'œil. L'espace interstitiel remet en question le concept même d'hybridité dans la mesure où il s'agit d'une zone de transition entre les contrastes, qui n'en sont donc pas, mais dont la continuité, qui est celle du vivant, est mise en évidence et investie, à même de desceller un paradigme de pensée, de réflexion et d'action autrement plus libérateur et fécond que les rhétoriques de la fin, du post-, de l'épuisement et de la disparition.

Khalil Khalsi est le lauréat 2016 du prix « Recherche au présent » pour la communication à l'origine du présent texte. Titulaire d'un Master de la Sorbonne Nouvelle, il a enseigné les théories de la culture à l'Université de Montréal, où il mène une thèse de doctorat, en cotutelle avec Paris-3, consacrée à l'étude du rêve et d'une poétique interstitielle à partir des œuvres de B. Gysin, S. Hedayat, I. al-Koni et A. Volodine. Il est notamment l'auteur d'un article intitulé « Sur la route avec l'animal en soi. La destinée selon Henry Bauchau » (Revue internationale Henry Bauchau, $\left.\mathrm{n}^{\circ} 7,2015\right)$. 


\section{Bibliographie}

Agier, Michel. La Condition cosmopolite. La Découverte, 2013.

Serres, Michel. Atlas. Julliard, 1994.

Esposito, Roberto. Third Person. Traduit par Zakiya Hanafi, Polity press, 2012.

Fleury, Cynthia. Les Irremplaçables. Gallimard, 2015.

Jameson, Fredric. Archéologies du futur, Tome 1: Le désir nommé utopie. Traduit par Nicolas Vieillescazes et Fabien Ollier. M. Milo, 2007.

---. "Utopia as Method, or the Uses of the Future." Utopia/Dystopia (éd. Michael D. Gordin, Helen Tilley, et Gyan Prakash). Princeton University Press, 2010, pp. 21-44.

Jullien, François. «L'écart et l'entre », 15 fév. 2012, halshs.archives-ouvertes.fr, Web. 8 nov. 2016.

Rabaté, Dominique. Désirs de disparaître. Tangence, 2015.

Volodine, Antoine. Le Post-exotisme en dix leçons, leçon onze. Gallimard, 1998.

---. « Écrire en français une littérature étrangère ». Chaö̈, 6 (2002), Web. 8 nov. 2016.

---. Bardo or not Bardo. 2004. Éd. du Seuil, 2005.

---. "À la frange du réel." Défense et illustration du post-exotisme en vingt leçons : avec Antoine Volodine. VLB éd., 2008.

---. Le Port intérieur. 1995. Minuit, 2010.

Westphal, Bertrand. La Géocritique, Minuit, 2007.

\footnotetext{
${ }^{1}$ « Je cherche à explorer et à représenter une culture non pas relativement, mais ABSOLUMENT étrangère. » (Volodine, "Écrire en français...")

${ }^{2} \mathrm{Au}$ sens étymologique : du grec metaphorai, « transport ».

${ }^{3}$ Les personnages post-exotiques « effectuent des allées et venues d'une âme à une autre, ils vagabondent d'un songe à un autre, ils glissent d'un univers à un autre. » (Volodine, Le Post-exotisme... 75)
} 